\title{
Deeply Virtual Compton Scattering in Color Dipole Formalism
}

\author{
Magno V. T. Machado \\ Universidade Federal do Pampa - UNIPAMPA, Campus Bagé. \\ Rua Carlos Barbosa, CEP 964000-970. Bagé, RS, Brazil
}

Received on 31 August, 2006

\begin{abstract}
In this contribution we summarize recent investigations on the deeply virtual Compton Scattering (DVCS) within the color dipole approach. The color dipole cross section is implemented through the phenomenological saturation model. The role played by its QCD evolution and skewedness effects in the DVCS cross section are discussed. The results are compared with the recent H1 and ZEUS Collaborations data. The skewing factor, defined as the ratio of the imaginary parts of the amplitudes $\operatorname{Im} \mathcal{A}\left(\gamma^{*} p \rightarrow \gamma^{*} p\right) / \operatorname{Im} \mathcal{A}\left(\gamma^{*} p \rightarrow \gamma p\right)$ can be extracted from the data using recent DVCS and the inclusive inelastic cross section measurements at DESY-HERA. We report on this experimental extraction and compare the results to the theoretical predictions for NLO QCD and the color dipole approach.
\end{abstract}

Keywords: Deeply virtual Compton scattering; High energy physics; Small- $x$ physics; Saturation models

\section{INTRODUCTION}

The study of hard exclusive reactions in the Bjorken limit is crucial to obtain information on parton dynamical correlations in the nucleon.It is well known that the cross section of hard scattering processes can be described as the convolution of parton distributions (PDFs) and the cross sections of hard subprocesses computed at parton level using perturbative QCD. The usual PDFs, obtained from inclusive experimental data, is the diagonal element of an operator in the Wilson operator product expansion (OPE). On the other hand, there is a set of exclusive reactions which are described by the off-diagonal elements of the density matrix, where the momentum, helicity or charge of the outgoing target are not the same as those of the corresponding incident particle. Examples of such reactions are the virtual photon Compton scattering (DVCS) $\left(\gamma^{*} p \rightarrow \gamma p\right)$ and the vector meson electroproduction $\left(\gamma^{*} p \rightarrow V p\right)$. In these cases, the difference with the inclusive case is the longitudinal components of the incoming and outgoing proton momentum, which depend on the photon virtuality $Q^{2}$ and the $\gamma^{*} p$ center of mass energy $W$. The recent data from DESY ep collider HERA on exclusive diffractive virtual Compton process [1-3] at large $Q^{2}$ becomes an important source to study the partons, in particular gluon, inside the proton for non-forward kinematics and its relation with the forward one (see Fig. I). A considerable interest of the DVCS process comes from the particular access it gives to these generalized parton distributions (GDP) through the interference term with the Bethe-Heitler process. On the other hand, recently the color dipole formalism has been provided a simultaneous description of photon induced process. The inclusive deep inelastic reaction and the photon diffractive dissociation has been successfully described and the study of other exclusive process as DVCS is an important test of the color dipole approach. In this contribution one summarizes the studies in Refs. $[4,5]$, where one applies the successful saturation model [6] to the DVCS process. The model interpolates between the small and large dipole configurations and has its parameters obtained from an adjust to small $x$ HERA data. Moreover, its QCD evolution has been recently computed [7], which improves the high $Q^{2}$ data description. Moreover, we present the recent results on the experimental extraction of the skewing correction factor $R$ [8], which it was performed by computing the ratio of the imaginary parts of the amplitudes for the DIS and DVCS reactions. This knowledge enables us to gain insight into the skewedness correction to the non-forward observables. In what follows, we present the main formulae for DVCS process in the color dipole formalism, introducing the phenomenological saturation model and its comparison with experimental data. Finally, one presents the extraction of the skewing factor from experimental results.

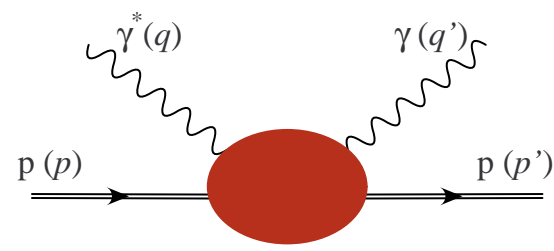

FIG. 1: Amplitude level diagram for DVCS process.

\section{DVCS IN THE COLOR DIPOLE PICTURE}

Based on the color dipole framework, the DVCS process can be seen as a succession in time of three factorisable subprocesses: i) the photon fluctuates in a quark-antiquark pair, ii) this color dipole interacts with the proton target, iii) the quark pair annihilates in a real photon. As usual, the kinematic variables are the c.m.s. energy squared $s=W_{\gamma p}^{2}=(p+q)^{2}$, where $p$ and $q$ are the proton and the photon momenta respectively, the photon virtuality squared $Q^{2}=-q^{2}$ and the Bjorken scale $x=Q^{2} /\left(W_{\gamma p}^{2}+Q^{2}\right)$. The DVCS imaginary part of the amplitude at zero momentum transfer reads as,

$$
\begin{aligned}
\operatorname{Im} \mathcal{A}\left(s, Q^{2}\right) & =\int_{0}^{1} d z \int d^{2} r \Psi_{T}^{*}\left(z, r, Q_{1}^{2}=Q^{2}\right) \Psi_{T}\left(z, r, Q_{2}^{2}=0\right) \\
& \times \sigma_{d i p}\left(\tilde{x}, r^{2}\right)
\end{aligned}
$$




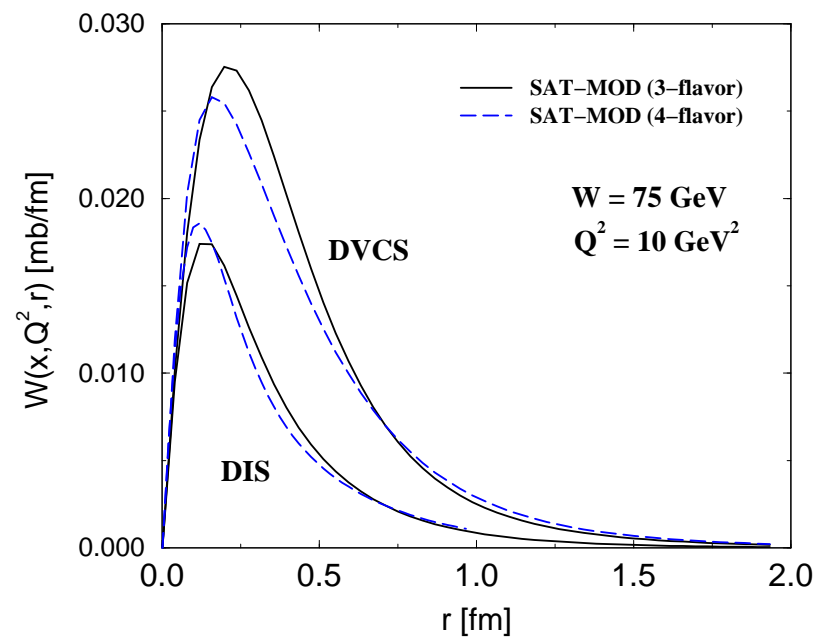

(a)

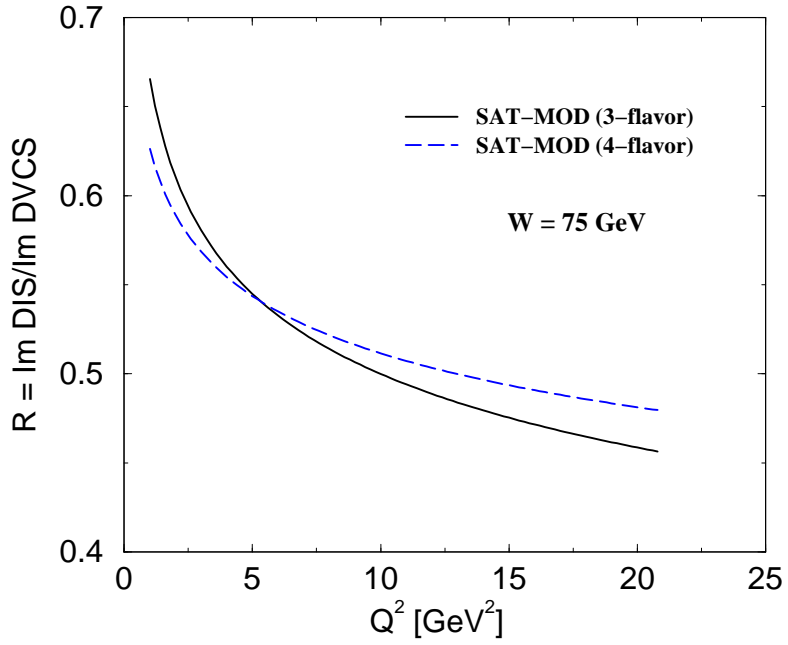

(b)

FIG. 2: (a) Comparison between the profile function $W\left(r, Q^{2}\right)$ as a function of the dipole size $r$ for DVCS and inclusive DIS processes at $Q^{2}=10 \mathrm{GeV}^{2}$. (b) The ratio of the imaginary parts of the DIS and DVCS amplitudes as a function of $Q^{2}$ at $W=75 \mathrm{GeV}$.

where $\sigma_{d i p}\left(\tilde{x}, r^{2}\right)$ is the dipole cross section, which depends on the scaling variable $\tilde{x}=\frac{Q^{2}+4 m_{f}^{2}}{\left(W_{\gamma p}^{2}+Q^{2}\right)}$ and dipole size, $r$. The product of the photon wavefunctions (transverse polarization) is given by,

$$
\begin{aligned}
\Psi_{T}^{*} \Psi_{T} & =\frac{6 \alpha_{\mathrm{em}}}{4 \pi^{2}} \sum_{f} e_{f}^{2}\left[z^{2}+(1-z)^{2}\right] \varepsilon_{1} K_{1}\left(\varepsilon_{1} r\right) \varepsilon_{2} K_{1}\left(\varepsilon_{2} r\right) \\
& +m_{f}^{2} K_{0}\left(\varepsilon_{1} r\right) K_{0}\left(\varepsilon_{2} r\right)
\end{aligned}
$$

where $\varepsilon_{1,2}^{2}=z(1-z) Q_{1,2}^{2}+m_{f}^{2}$. The quark mass, $m_{f}$, plays the role of a regulator as $Q^{2} \rightarrow 0$. The relative contributions from dipoles of different sizes can be analyzed with the weight (profile) function,

$W\left(r, Q^{2}\right)=\int_{0}^{1} d z r \Psi_{T}^{*}\left(z, r, Q_{1}^{2}\right) \Psi_{T}\left(z, r, Q_{2}^{2}\right) \sigma_{d i p}\left(\tilde{x}, r^{2}\right)$.

In the DIS process the contribution of large dipole configurations is observed to diminish in a sizable way as the virtualities increase. However, as shown in Fig. (2-a), the DVCS profile selects larger dipole sizes in contrast to the inclusive DIS case, even at relatively large $Q^{2}$ (similar result has been obtained in Ref.[9]). The calculation was performed using the saturation model [6] for both three and four-flavor analysis. The inclusion of the charm content gives a lower normalization for the profile and by consequence for the total cross section. The impact of the charm is smaller in the inclusive DIS case than in DVCS process, confirming that DVCS is more sensitive to the non-perturbative (soft) content of the scattering process. Moreover, in order to estimate the importance of the skewing effect, we calculate the ratio between the imaginary parts of the forward $t=0$ amplitudes for DIS and DVCS, $\mathrm{R}=\operatorname{Im}$ DIS / Im DVCS. As shown in Fig. (2-b) our result presents values slightly above those from an aligned jet model analysis in Ref. [10] and below those from the dipole analysis in Ref. [9].
The final expression for the DVCS cross section is written as,

$$
\sigma\left(\gamma^{*} p \rightarrow \gamma p\right)=\frac{\left[\operatorname{Im} \mathcal{A}\left(s, Q^{2}, t=0\right)\right]^{2}}{16 \pi b}\left(1+\eta^{2}\right),
$$

where $b$ is the $t$ slope parameter and comes from a simple exponential parameterization and $\eta$, the ratio between the real to imaginary part, is computed according to the dispersion relations (more details in Ref. [4]).

\section{CROSS SECTION COMPARISON TO DATA}

In what follows we present the results for the phenomenological saturation models and also its version considering QCD evolution [7] (labeled BGBK), giving the dipole cross section gluon dependent. In Fig. II (from H1 Coll. paper [3]) one compares their prediction with the existing measurement from $\mathrm{H} 1$ and ZEUS Collaborations [1-3]. The color dipole prediction from Donnachie-Dosh [11] is also presented for the sake of comparison. For the color dipole models, we take a fixed slope value $b=7 \mathrm{GeV}^{-2}$ in Eq. (3). The average experimental value is $b \simeq 6 \mathrm{GeV}^{-2}$ [3]. The reason is to single out the importance of $Q^{2}$-evolution from BGBK without introduce any additional background coming from the slope. It should be noticed that both saturation models are equivalent in the description of inclusive DIS data in the virtuality range considered here. As the dependencies of $B$ on $Q^{2}$ and $W$ are not well constrained from experimental measurements, the normalization of the theoretical prediction is basically free.

Although there is a little difference of normalization between $\mathrm{H} 1$ and ZEUS measurements, which makes difficult to set an overall $B$ value for all measurements, the behavior on $Q^{2}$ and $W$ (see Ref. [3]) is well reproduced. On the other hand, for $Q^{2}>40 \mathrm{GeV}^{2}$ our prediction still underestimates the experimental data. This change of behavior in the $Q^{2}$ shape 


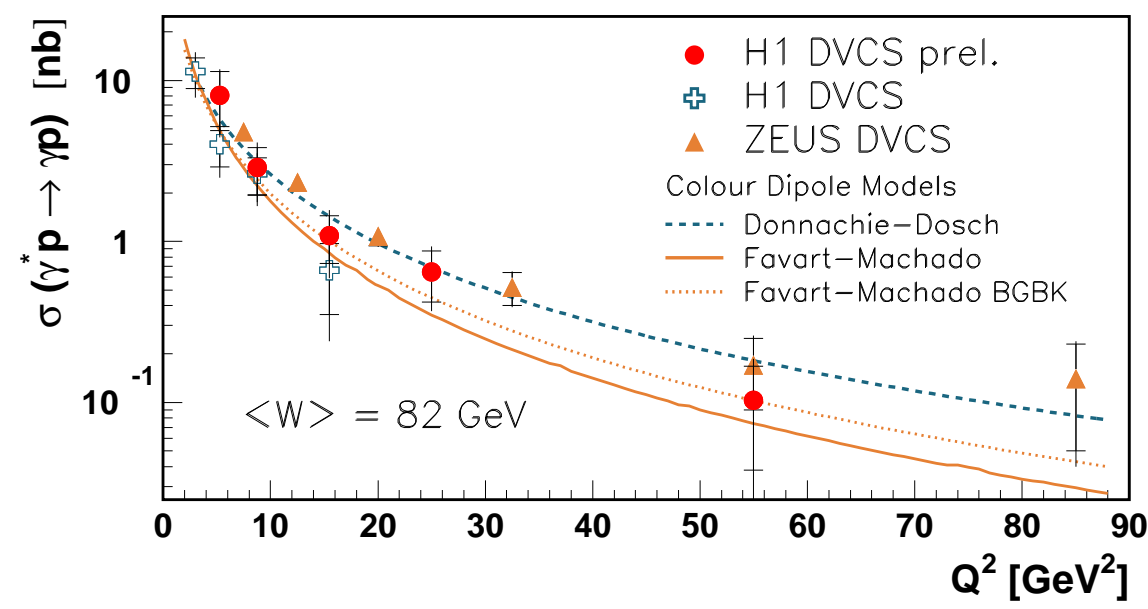

FIG. 3: The photon level DVCS cross section as a function of virtuality $Q^{2}$ at $W=75 \mathrm{GeV}$. Data are compared to the present prediction, with and without (BGBK) QCD evolution (see text).

can indicate two situations: (a) the $B$ slope would diminish as increasing virtualities or; (b) some additional effect should appear at higher $Q^{2}$. In order to investigate the first hypothesis, we compute cross section using a $Q^{2}$ dependent slope, $b\left(Q^{2}\right)=8\left[1-0.15 \ln \left(Q^{2} / 2\right)\right] \mathrm{GeV}^{-2}$ (see Ref. [5] for details), in the range $2 \leq Q^{2} \leq 100 \mathrm{GeV}^{2}$. This gives $b \simeq 8$ $\mathrm{GeV}^{-2}$ at $Q^{2} \simeq 2 \mathrm{GeV}^{2}$ going to $b \simeq 3.3 \mathrm{GeV}^{-2}$ at $Q^{2} \simeq 100$ $\mathrm{GeV}^{2}$, which is consistent with rho meson electroproduction results. Concerning the second hypothesis, we have investigated two options: QCD evolution (using BGBK model) and skewedness effects. For the skewedness corrections, the ratio of off-forward to forward gluon distribution are are given explicitly by [12], $R_{g}\left(Q^{2}\right)=\frac{2^{2 \lambda+3}}{\sqrt{\pi}} \frac{\Gamma\left(\lambda+\frac{5}{2}\right)}{\Gamma(\lambda+4)}$, where $\lambda$ is the effective power on energy of the scattering amplitude. For our purpose the amplitude is multiplied by $R_{g}$, in order to estimate the size of the skewedness effects.

To compare the $Q^{2}$ dependence, we normalize all models to describe the ZEUS data point at the lowest $Q^{2}$ value. Further, we plot the ratio of each model to our baseline model SAT-MOD as a function of $Q^{2}$. Such a procedure allows a $Q^{2}$ dependence comparison independently of the normalization effect. These ratios are shown in Fig. IV, where the points (triangles-up) are the ratio of the ZEUS data to SAT-MOD including the error bars for the statistical (inner) and sum in quadrature of statistical and systematic (outer) uncertainties.

We verify that several models can account for the measured $Q^{2}$ dependence, which are not distinguishable with the present experimental precision. If the change in normalization is small for the inclusion of a $Q^{2}$ dependence in $b$, the effect is of the order of $12 \%$ for BGBK with respect to the basic SAT-MOD and of $40 \%$ for the skewedness effect (SKEW) and still larger when the different effect are combined $(60 \%$ for BGBK+SKEW). Therefore, these issues show clearly the importance of a precise measurement of the slope $b$. Such a measurement would already allow to discriminate among the different theoretical predictions with an amount of data comparable to the present ZEUS measurement.

\section{EXPERIMENTAL EXTRACTION OF SKEWING FACTOR}

Precision data are becoming available for hard scattering processes whose description requires knowledge of these offdiagonal (or skewed) parton distributions. The exclusive diffractive DVCS process at large $Q^{2}$ provides a comparatively clean procedure for extracting information on the gluons within the proton in a non-forward kinematic case. In recent years the study of Deep Inelastic lepton-proton Scattering (DIS) has produced detailed information of the proton structure in terms of Parton Distribution Functions (PDFs). The optical theorem states that the leading DIS process of a single virtual photon exchange can be viewed as identical to the forward elastic scattering of a virtual photon from the proton. This scattering, known as the forward Compton scattering process, is the same as a DIS process with its mirrored reaction and relates the total cross section $\sigma_{t o t}\left(\gamma^{*} p \rightarrow X\right)$ with the imaginary part of the forward amplitude $\operatorname{Im} A\left(\gamma^{*} p \rightarrow \gamma^{*} p\right)$.

The forward Compton scattering $\gamma^{*}(q) p(p) \rightarrow \gamma^{*}(q) p(p)$ can be generalized to the non forward case (at non-zero angles): $\gamma^{*}(q) p(p) \rightarrow \gamma^{(*)}\left(q_{2}\right) p\left(p_{2}\right)$ with $q_{2} \neq q$ and $p_{2} \neq p$ and being experimentally accessible through the so called Deeply Virtual Compton Scattering (DVCS) process, where a real photon is observed in the final state. In analogy with DIS which relates the imaginary part of the forward Compton amplitude and PDFs, in the DVCS process parton distributions are also used to parameterize the non-perturbative part of the interaction in the non-forward region. In this last case, the distributions have to be calculated in terms of non-diagonal, rather than diagonal, PDFs. They are commonly called Generalised Parton Distributions (GPDs). They reduce to the ordinary PDFs in the limit of zero skewedness, i.e., the forward limit. The most promising approach for GPD parameterizations relies on setting them equal to the forward PDFs using a forward model with suitably symmetrized input GPDs in the ERBL region constructed in order to satisfy polynomiality for the first two momenta [17]. These theoretical studies repro- 


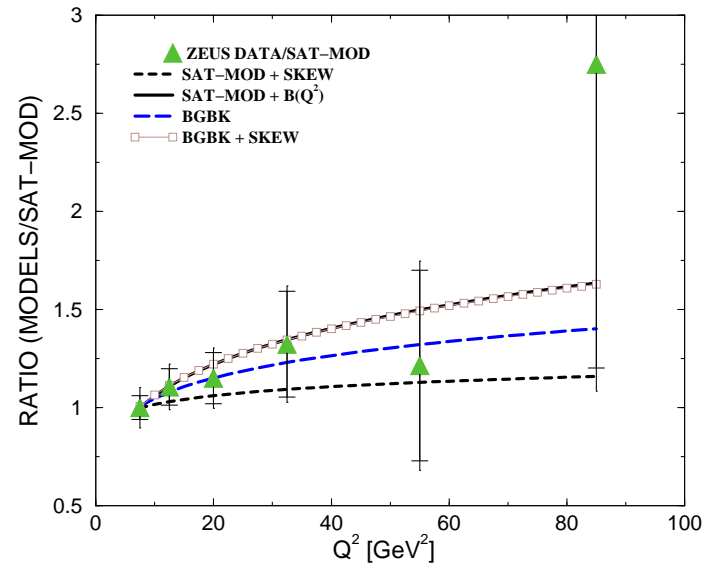

FIG. 4: The ratio MODELS/SAT-MOD as a function of $Q^{2}$ (see text for details).

duce satisfactorily the main features of DVCS data [3]. On the other hand, a simple and intuitive high energy formalism has recently been used to describe DVCS [4]. It is given by a color dipole picture, which allows extending in the studies of saturation physics at low virtualities of the incoming photon. This is an advantage in comparison with the pQCD formalism which is limited by the initial scale for QCD evolution to the order of $Q_{0} \gtrsim 1 \mathrm{GeV}$. Additionally, the roles played by QCD evolution and skewing effects have been recently shown in the scope of this formalism. The off-diagonal effect has been investigated through a simple phenomenological parameterization. The overall picture is in good agreement with the experimental data [5].

Lets now address the question of the extraction of the skewing correction using the available data on DIS and DVCS. This can be performed by computing the ratio of the imaginary parts of the amplitudes for these reactions. The skewing factor $R$ depends on the experimental measurements and therefore possess a small degree of model dependence. We, therefore, have the attractive possibility in determining its dependence upon $Q^{2}$ virtuality and upon $W$ energy. This knowledge will enable us to gain insight into the skewedness correction to the non-forward observables. Lets define a basic quantity giving an overall measurement of the skewing properties, which includes both the non-forward kinematics and the non-diagonal effects. Namely, we set the ratio between the imaginary parts of the DIS and DVCS (forward) scattering amplitudes at zero momentum transfer:

$$
R \equiv \frac{\left.\operatorname{Im} \mathcal{A}\left(\gamma^{*}+p \rightarrow \gamma^{*}+p\right)\right|_{t=0}}{\left.\operatorname{Im} \mathcal{A}\left(\gamma^{*}+p \rightarrow \gamma+p\right)\right|_{t=0}}
$$

where $t$ is the square of the four-momentum exchanged at the proton vertex.

The scattering amplitude for the DIS process can be directly obtained from the DIS cross section and experimentally measured at DESY-HERA, that is $\operatorname{Im} \mathcal{A}\left(\gamma^{*} p \rightarrow \gamma^{*} p\right) \sim$ $\sigma_{t o t}\left(\gamma^{*} p \rightarrow X\right)$. In fact, the DIS amplitude can be written down using the usual pQCD fits for the proton structure function, $\sigma_{\text {tot }}^{\gamma^{*} p}=\left(4 \pi^{2} \alpha / Q^{2}\right) F_{2}^{p}\left(x, Q^{2}\right)$. The DVCS scattering amplitude can be obtained from the recent measurements on the Deeply Virtual Compton Scattering cross section. In this case, the $t$ dependence of the amplitude can be assumed to be factorised out and parameterized as an exponential, $\propto \exp (-b|t|)$, and the total DVCS cross section can be related to the corresponding amplitude at $t=0$; as referred in Eq. (3). The typical contribution of the real part of amplitude for the kinematic window available at HERA is of the order of $20 \%$. In particular, to a good approximation, $\eta$ can be calculated using dispersion relations to $\eta \simeq \tan (\pi \lambda / 2)$, where $\lambda=\lambda\left(Q^{2}\right)$ is the effective power of the Bjorken $x$ dependence of the imaginary part of the amplitude. For an estimate of the real part contribution we use the effective power for the inclusive deep inelastic reaction taken from Ref. [13].

Considering the calculation discussed above, we can rewrite the skewing factor as a function of the total cross sections for DIS and DVCS. The factor reads as,

$R=\frac{\sigma\left(\gamma^{*} p \rightarrow X\right) \sqrt{\left(1+\eta^{2}\right)}}{4 \sqrt{\pi b \sigma\left(\gamma^{*} p \rightarrow \gamma p\right)}}=\frac{\sqrt{\pi^{3}} \alpha}{Q^{2}} \frac{F_{2}^{p}\left(x, Q^{2}\right) \sqrt{\left(1+\eta^{2}\right)}}{\sqrt{b \sigma\left(\gamma^{*} p \rightarrow \gamma p\right)}}$.

Main theoretical uncertainties come from the $b$ slope and from the estimate of the real part contribution. In our further calculation, one uses the $b$ value extracted from the recent measurements of the $t$-dependence of DVCS data.

To extract the factor $R$ factor, Eq. (5), we use recent DVCS measurements at HERA [2, 3] and the DIS cross cross section is obtained using the pQCD fits of the $F_{2}^{p}$ structure function from Ref. [14]. The factor $R$ is shown as a function of $Q^{2}$ in Fig. 5-a and as a function of energy $W$ in Fig. 5-b. The inner error bars represent the statistical error. The full error bars is the quadrature sum of the statistical, systematic and normalization (precision of the $b$ measurement of H1) uncertainties. A $Q^{2}$ dependence is observed, decreasing from $R \simeq 0.7$ for $Q^{2} \simeq 2 \mathrm{GeV}^{2}$ down to $R \simeq 0.3$ for $Q^{2} \simeq 85 \mathrm{GeV}^{2}$. Qualitatively, the data seem to be consistent with a dependence like $R \propto 1 / \log Q^{2}$. An almost flat $W$ dependence is observed within the present precision. This feature can be easily understood by inspecting Eq. (5), since the $W$ dependence of both the DIS and DVCS cross section is power-like having a proportional effective power. Namely, $\sigma_{\text {DIS }} \propto W^{2 \lambda}$ and $\sigma_{\text {DVCS }} \propto W^{4 \lambda}$. The mean value $R \simeq 0.5$ is consistent with its early theoretical estimates using the aligned jet model [10] and the color dipole saturation model [4].

Lets compare the results extracted from experimental data to different theoretical predictions. First, we contrast them with the perturbative QCD approach at NLO accuracy. This prediction is dependent on the Generalised Parton Distributions (GPD), which have been parameterized in Ref. [17] and applied to describe the recent DVCS data $[15,16]$. The second theoretical approach $[4,5]$ is given by the color dipole approach, where the basic building blocks are the photon wavefunctions and the dipole cross section. The non-forward kinematics is encoded by the wavefunctions whereas the off- 


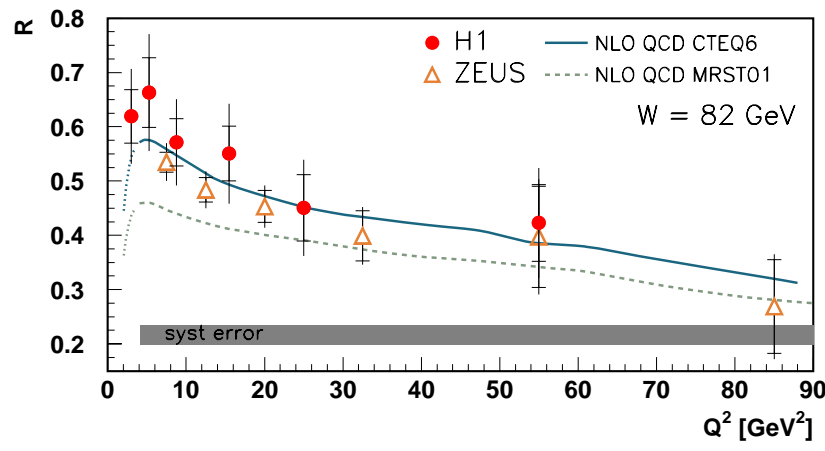

(a)

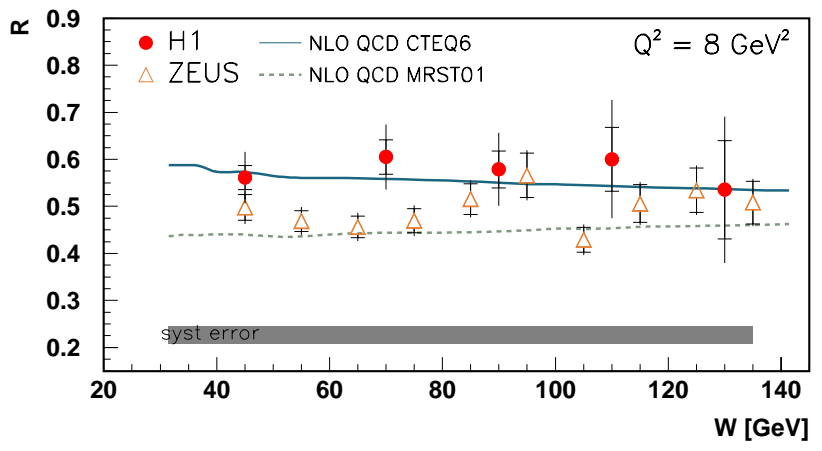

(b)

FIG. 5: (a) The skewing factor $R$ as a function of $Q^{2}$ at $W=82 \mathrm{GeV}$. The points correspond to the $R$ extraction applied to DVCS measurements of H1 (bullets) and ZEUS (triangles). The curves represent $R$ extracted from Freund et al. prediction based on MRST 2001 and CTEQ6 PDFs. (b)The skewing factor $R$ as a function of $W$ at $Q^{2}=8 \mathrm{GeV}^{2}$.

diagonal effects can be built-in in the parameterizations for the dipole-target cross section.

The DVCS cross section has been calculated at NLO in perturbative QCD by Freund and McDermott $[15,16]$ using two different GPD parameterizations [17]. The MRST2001 and CTEQ6 parameterizations of the PDFs are used in the DGLAP region and polynomial form is used in the ERBL region, ensuring a smooth continuation between the two regions. Both the skewing $(\xi)$ and the $Q^{2}$ dependence are generated dynamically. The $t$ dependence is factorised out and assumed to be $e^{-b|t|}$. In its original form, the approach above used a $Q^{2}$-dependent $b$ slope. We have verified that the recent data on DVCS [3] can be reasonably described using a fixed $b$ slope.

Applying the same method presented here (i.e. using the same $F_{2}^{p}$ ) for the inclusive cross section, the values obtained are shown as a function of $Q^{2}$ in Fig. 5-a and as a function of $W$ in Fig. 5-b. The value of $b=6.02 \pm 0.35 \pm 0.39 \mathrm{GeV}^{-2}$ measured by $\mathrm{H} 1$ [3] has been used in all predictions. The error on the $b$ value is not applied to theoretical predictions as it is already included in the total error of the data points. A good agreement between these predictions and the data points is found, describing well the absolute value of $R$ and its kinematic dependences. The NLO calculation presents no $W$ dependence. The result normalization is strongly dependent on the choice for the gluon distribution and the deviations seem to be amplified at lower $Q^{2}$. Concerning the behavior on $Q^{2}$, it seems that for $Q^{2}$ values below $\simeq 4 \mathrm{GeV}^{2}$ the DGLAP evolution starting at $Q_{0}^{2}=1 \mathrm{GeV}^{2}$ has too little phase space to fully generate the gluon distributions (dotted part of the curves in Fig. 5-a). The underestimation of $R$ for virtualities below 4 $\mathrm{GeV}^{2}$ may be due to a larger value for the DVCS cross section calculated at NLO where the gluon distribution is underestimated as an effect of the too small phase space for evolution.

In the color dipole approach, as referred before, the imaginary part of the DIS (or DVCS) amplitude at $t=0$ is expressed in the simple way [4]. The non-forward kinematics for DVCS is encoded in the color dipole approach through the different weight coming from the photon wavefunctions in Eq. (1). The off-diagonal effects, which affect the gluon and quark distributions in the pQCD approaches, should be included in the parameterization of the dipole cross section. At the present stage of the development of the color dipole formalism, we have no accurate theoretical arguments on how to compute skewedness effects from first principles. A consistent approach would be to compute the scattering amplitude in the non-forward case, since the non-forward photon wave function has been recently obtained in Ref. [18]. In this case, the dipole cross section, $\sigma_{\text {dip }}\left(x_{1}, x_{2}, r, \vec{\Delta}\right)$, depends on the light cone momenta $x_{1}$ and $x_{2}$ carried by the exchanged gluons, respectively, and on the total transverse momentum transfer $\vec{\Delta}$. In this case, additional information about the dependence upon $\vec{\Delta}$ is needed for the QCD Pomeron and proton impact factor. The forward dipole cross section is recovered at $x_{1}=x_{2}$ and $\vec{\Delta}=0$.

In Ref. [5] an estimate of the skewedness for the dipole cross section has been performed using the approximation of Ref. [12] where the ratios of off-diagonal to diagonal parton distributions are computed. The ratio is larger for singlet quarks than for gluons. In the color dipole picture, the DVCS cross section is driven mainly by gluonic exchanges. In our numerical computations, we use $\lambda=\lambda\left(Q^{2}\right)$ as obtained from the DVCS scattering amplitude and the skewedness effect is given by multiplying the original (without skewedness) total cross section by the factor $R_{g}^{2}\left(Q^{2}\right)$. A different implementation of the skewedness correction is to use the approximation $\tilde{x}=0.41 x_{\mathrm{Bj}}$, which produces the same effects as for $R_{g}$ at the presently covered kinematic range and precision of the measurement. This different procedure is particularly suitable for dipole cross sections which are parameterized by using a gluon distribution, namely $\sigma_{d i p} \propto r^{2} x g\left(x, c / r^{2}\right)$.

Using this color dipole prediction (with DGLAP evolution and off-diagonal skewing factor $R_{g}$ ), one can again extract the $R$ factor, as shown as a function of $Q^{2}$ in Fig. 6-a and as a function of $W$ in Fig. 6-b. A good agreement with points extracted from the data is found. The curve obtained without applying the off-diagonal skewing factor $R_{g}$ is also presented, 


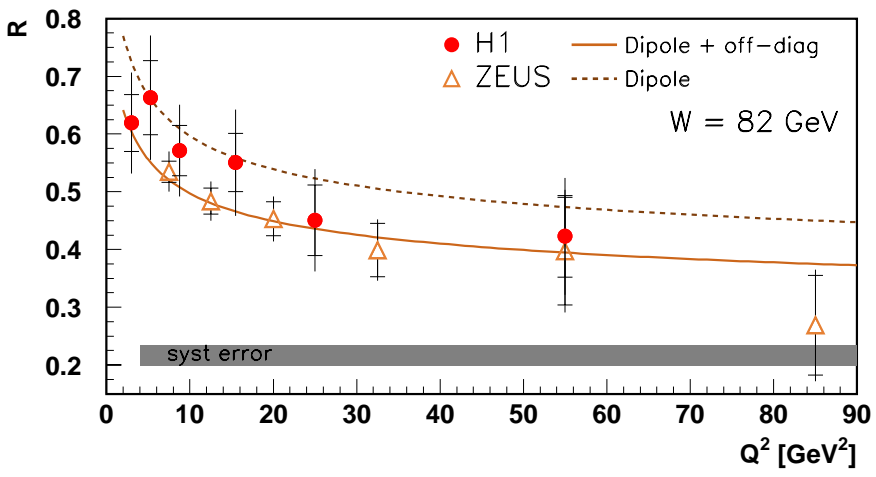

(a)

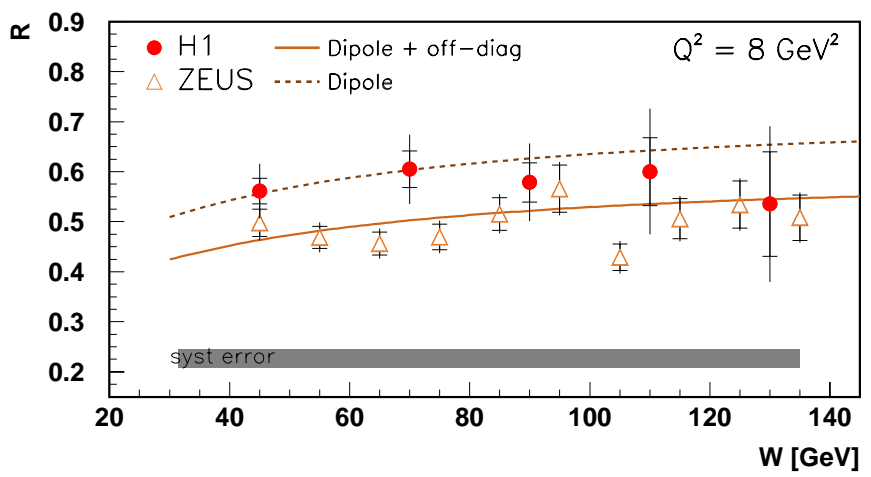

(b)

FIG. 6: (a) The skewing factor $R$ as a function of $Q^{2}$ at $W=82 \mathrm{GeV}$. The curves represent the theoretical predictions of the color dipole approach (see text) with (solid) and without (dashed) applying the off-diagonal skewing factor $R_{g}$. (b) The skewing factor $R$ as a function of $W$ at $Q^{2}=8 \mathrm{GeV}^{2}$

enabling one to isolate the two effects: the non-forward kinematic and the off-diagonal gluon distributions. Furthermore, the off-diagonal effects contribute mostly for the overall normalization of the skewing factor whereas the behavior on $Q^{2}$ seems to be driven by the non-forward kinematic effect. This prediction exhibits a moderate increasing of $R$ with $W$ which is not present in the NLO prediction.

\section{SUMMARY}

We summarize the investigations on DVCS within the color dipole approach. The color dipole cross section is implemented through the phenomenological saturation model. The role played by its QCD evolution and skewedness effects in the DVCS cross section are discussed. The results are compared with the recent $\mathrm{H} 1$ and ZEUS Collaborations data and the description is in good agreement with the experimental measurements. The skewing factor $R$, defined as the ration of the imaginary parts of the DIS and DVCS amplitudes has been extracted from experimental data for the first time. In its determination one uses the recent DVCS and the inclusive inelastic cross section measurements at DESY-HERA. The main theoretical uncertainties come from the $b$ slope and from the estimate for the real part contribution. One founds a $Q^{2}$ dependence qualitatively consistent with the form $R \propto 1 / \log Q^{2}$. No $W$ dependence is observed within the current precision. The mean value $R \simeq 0.5$ is consistent with previous theoretical estimates in the jet aligned model and color dipole picture. The extracted $R$ is contrasted to theoretical predictions of NLO QCD and color dipole approaches.

\section{Acknowledgments}

The results presented in this contribution were obtained in collaboration with L. Favart (H1 Coll./ULB) and L. Schoeffel (CEA/Saclay), who are gratefully acknowledged.
[1] C. Adloff et al. [H1 Collaboration], Phys. Lett. B 517 (2001) 47.

[2] S. Chekanov et al. [ZEUS Collaboration], Phys. Lett. B 573, 46 (2003).

[3] C. Aktas et al. [H1 Collaboration], Eur. Phys. J. C 44, 1-11 (2005).

[4] L. Favart and M. V. T. Machado, Eur. Phys. J. C 29 (2003) 365.

[5] L. Favart and M. V. T. Machado, Eur. Phys. J. C 34 (2004) 429.

[6] K. Golec-Biernat and M. Wusthoff, Phys. Rev. D 59 (1999) 014017.

[7] J. Bartels, K. Golec-Biernat and H. Kowalski, Phys. Rev. D 66 (2002) 014001

[8] L. Favart, M. V. T. Machado and L. Schoeffel, arXiv:hep$\mathrm{ph} / 0511069$.

[9] M. McDermott, R. Sandapen and G. Shaw, Eur. Phys. J. C 22 (2002) 655
[10] L. L. Frankfurt, A. Freund and M. Strikman, Phys. Rev. D 58, 114001 (1998).

[11] A. Donnachie and H. G. Dosch, Phys. Lett. B502 (2001) 74.

[12] A. G. Shuvaev et al., Phys. Rev. D 60, 014015 (1999).

[13] C. Adloff et al. [H1 Collaboration], Phys. Lett. B 520, 183 (2001), [hep-ex/0108035].

[14] C. Adloff et al. [H1 Collaboration], Eur. Phys. J. C21 (2001) 33-61.

[15] A. Freund and M.F. McDermott, Phys. Rev. D 65 (2002) 091901.

[16] A. Freund and M.F. McDermott, Phys. J. C 23 (2002) 651-674.

[17] A. Freund, M.F. McDermott and M. Strikman, Phys. Rev. D 67 (2003) 036001.

[18] J. Bartels, K. Golec-Biernat and K. Peters, Acta Phys. Polon. B 34, 3051 (2003). 